\title{
Two-dimensional boson-fermion mixtures
}

\author{
A. L. Subaşi, ${ }^{1}$ S. Sevinçli, ${ }^{1, *}$ P. Vignolo, ${ }^{2}$ and B. Tanatar ${ }^{1}$ \\ ${ }^{1}$ Department of Physics, Bilkent University, Bilkent, Ankara 06800, Turkey \\ ${ }^{2}$ Institut Non Linéaire de Nice, CNRS, Université de Nice-Sophia Antipolis, 1361 route de Lucioles, O6560 Valbonne, France
}

(Received 13 May 2009; published 30 June 2009)

\begin{abstract}
Using mean-field theory, we study the equilibrium properties of boson-fermion mixtures confined in a harmonic pancake-shaped trap at zero temperature. When the modulus of the $s$-wave scattering lengths are comparable to the mixture thickness, two-dimensional scattering events introduce a logarithmic dependence on density in the coupling constants, greatly modifying the density profiles themselves. We show that, for the case of a negative boson-fermion three-dimensional $s$-wave scattering length, the dimensional crossover stabilizes the mixture against collapse and drives it toward spatial demixing.
\end{abstract}

DOI: 10.1103/PhysRevA.79.063632

PACS number(s): 03.75.Hh, 03.75.Ss, 64.75.Cd

\section{INTRODUCTION}

Fermionic atomic gases were brought together with bosonic atoms to quantum degeneracy in several alkali-metal atom mixtures, such as ${ }^{7} \mathrm{Li}-{ }^{6} \mathrm{Li}[1,2],{ }^{23} \mathrm{Na}-{ }^{6} \mathrm{Li}[3],{ }^{87} \mathrm{Rb}-{ }^{40} \mathrm{~K}$ [4-6], and very recently in a mixed gas of ytterbium ( $\mathrm{Yb}$ ) isotopes, ${ }^{174} \mathrm{Yb}-{ }^{173} \mathrm{Yb}[7]$. The boson-fermion (BF) coupling strongly affects the equilibrium properties of the mixture and can drive quantum phase transitions, as collapse [6] in the presence of attractive BF interaction, or spatial demixing, as recently observed in the context of three-dimensional (3D) atomic fermion-molecular boson mixtures [8,9], where the strong interspecies repulsion leads to phase separation.

Such mixtures can be realized from an imbalanced twocomponent Fermi gas $\left({ }^{40} \mathrm{~K}-{ }^{40} \mathrm{~K}\right.$ or ${ }^{6} \mathrm{Li}-{ }^{6} \mathrm{Li}$ mixture) where all minority fermions become bound as bosons and form a Bose-Einstein condensate (BEC). Although imbalanced Fermi gases allowed us to observe spatial phase separation between bosonic dimers and fermions, the advantage of a two atomic species BF mixture is that boson-boson (BB) and $\mathrm{BF}$ interactions can be driven independently and that one can access attractive BF interactions $[10,11]$.

The structure and the stability of trapped BF mixtures were studied in three dimensions by using the Thomas-Fermi (TF) approximation for the bosonic component $[12,13]$ and by using a modified Gross-Pitaevskii equation (GPE) for the bosons which self-consistently includes the mean-field interaction generated by the fermionic cloud $[14,15]$. Effects of the geometry induced by the trap deformation were studied in the Thomas-Fermi regime in a quasi-3D (Q3D) limit, i.e., when collisions can be still considered as three dimensional [16]. Such a simple model predicts, in a pancake-shaped trap, that the stability of the mixture depends only on the scattering length and the transverse width of the cloud. One should expect, in a true dimensional crossover, namely, including dimensional effects in scattering events, that the mixture stability depends critically on the energy and thus on the number of particles.

The dimensional crossover from a $3 \mathrm{D}$ to a twodimensional (2D) trapped mixture may be studied in the ex-

\footnotetext{
*Present address: Max-Planck-Institut für Physik Komplexer Systeme, Nöthnitzer Str. 38, 01187 Dresden, Germany.
}

periments by flattening magnetic or dipolar confinement [17], or by trapping atoms in specially designed pancake potentials, as rotating traps [18], gravito-optical surface traps [19], rf-induced two-dimensional traps [20], or in onedimensional lattices [21] where a 3D gas can be split in several independent disks.

In the limit where scattering events are bidimensional, it is well known that a hard-core boson gas shows very different features from its 3D counterpart. In three dimensions, particle interactions can be described by the zero-momentum and zero-energy limit of the $T$ matrix, leading to a constant coupling parameter. In two dimensions, the $T$ matrix vanishes at low momentum and energy [22,23] and the firstorder contribution to the coupling is obtained by taking into account the many-body shift in the effective collision energy of two-condensate atoms [24,25]. This leads to an energydependent coupling parameter that greatly affects the equilibrium and the dynamical properties of the gas [26,27].

In this paper we study the equilibrium properties of a mixture of condensed bosons and spin-polarized fermions through the dimensional crossover from three to two dimensions, by following the procedure outlined by Roth [14] for the 3D mixture. We neglect fermion-fermion interactions and we include BF $s$-wave interaction self-consistently in a suitably modified GPE for the bosons. For the case of BF repulsive interaction, the increasing anisotropy softens the repulsion, and a quasi-3D spatially demixed mixture is mixed in quasi-2D (Q2D). For the case of BF attractive interactions, the dimensional crossover acts as a Feshbach resonance and induces repulsive interactions, so that a Q3D mixture near collapse can be driven toward spatial demixing in Q2D. In the strictly $2 \mathrm{D}$ regime the results depend on the model that one assumes for the bidimensional scattering lengths.

The paper is organized as follows. In Sec. II we introduce the theoretical mean-field model for the description of ground-state density profiles of the BF mixture. The models for the coupling through the dimensional crossover are outlined in Sec. III. The density profiles obtained for a ${ }^{6} \mathrm{Li}-{ }^{7} \mathrm{Li}$ and ${ }^{40} \mathrm{~K}-{ }^{87} \mathrm{Rb}$ mixtures are shown in Sec. IV. Section $\mathrm{V}$ offers a summary and some concluding remarks.

\section{MEAN-FIELD MODEL FOR THE DENSITY PROFILES}

We consider a BF mixture in a $2 \mathrm{D}$ geometry, with respective particle numbers $N_{B}$ and $N_{F}$, confined in harmonic trap 
potentials $V_{B, F}=\frac{1}{2} m_{B, F} \omega_{B, F}^{2} r^{2}$. Here $m_{B, F}$ is the boson (fermion) mass and $\omega_{B, F}$ is the radial trap frequency as seen by boson or fermion species. Within the mean-field approach, the total energy functional at $T=0$ is written as

$$
\begin{aligned}
E\left[\psi_{B}, \psi_{F}\right]= & \int d^{2} r\left\{\frac{\hbar^{2}}{2 m_{B}}\left|\nabla \psi_{B}\right|^{2}+V_{B}(r)\left|\psi_{B}\right|^{2}+\frac{1}{2} g_{B B}\left|\psi_{B}\right|^{4}\right\} \\
& +\int d^{2} r\left\{T_{F}+V_{F}(r)\left|\psi_{F}\right|^{2}\right\}+\int d^{2} r g_{B F}\left|\psi_{B}\right|^{2}\left|\psi_{F}\right|^{2},
\end{aligned}
$$

where $\psi_{B, F}$ is the ground-state wave function of bosons and fermions, respectively. From the above, boson species are in the condensed state and fermion species is assumed to be spin polarized and its kinetic energy is written within the Thomas-Fermi-Weizsacker approximation as [28,29]

$$
T_{F}=\frac{\hbar^{2}}{m_{F}}\left(\pi n_{F}^{2}+\frac{\lambda_{W}}{8} \frac{\left|\nabla n_{F}\right|^{2}}{n_{F}}\right),
$$

where $n_{F}=\left|\psi_{F}\right|^{2}$ is the fermion density and the Weizsacker constant is $\lambda_{W}=1 / 4$. Normalization conditions for $N_{B}$ bosons and $N_{F}$ fermions read $N_{B}=\int d^{2} r\left|\psi_{B}\right|^{2}$ and $N_{F}=\int d^{2} r\left|\psi_{F}\right|^{2}$. The interaction couplings between the bosons and between bosons and fermions are denoted by $g_{B B}$ and $g_{B F}$, respectively. One notable difference between the forms of the energy functional given above and that in three dimensions is that the $\mathrm{BB}$ and the $\mathrm{BF}$ interaction strengths are in general density dependent in contrast to the situation in three dimensions. More specifically, in three dimensions, the interaction strengths are proportional to the scattering lengths $a_{B B}$ and $a_{B F}$, whereas in two dimensions as we shall explain below they depend on the density or equivalently the chemical potential. The Euler-Lagrange equations for the mixture read [29]

$$
\left\{-\frac{\hbar^{2}}{2 m_{B}} \nabla^{2}+V_{B}+g_{B B}\left|\psi_{B}\right|^{2}+g_{B F}\left|\psi_{F}\right|^{2}-\mu_{B}\right\} \psi_{B}=0
$$

and

$$
\left\{-\frac{\hbar^{2}}{2 m_{F}} \lambda_{W} \nabla^{2}+V_{F}+\frac{\hbar^{2}}{m_{F}} 2 \pi\left|\psi_{F}\right|^{2}+g_{B F}\left|\psi_{B}\right|^{2}-\mu_{F}\right\} \psi_{F}=0,
$$

in which we have introduced the chemical potentials $\mu_{B, F}$ for bosons and fermions. The above equations of motion are obtained by functional differentiation from $E\left[\psi_{B}, \psi_{F}\right]$ neglecting the higher-order terms involving $\delta g / \delta \psi_{B, F}$, which is valid in the dilute gas limit $n_{B} a_{B B}^{2} \ll 1$ and $n_{F} a_{B F}^{2} \ll 1$. The dilute gas conditions above further maintain that beyond mean-field corrections are not called for. They can become notable when $a_{B B}, a_{B F}$ and/or $N_{B}, N_{F}$ are large for fixed trap frequencies. For the systems under consideration, we have chosen the parameters appropriately and verified by numerical calculations, so that $n_{B} a_{B B}^{2}, n_{F} a_{B F}^{2} \ll 1$. Therefore, in the examples we shall discuss subsequently that the beyond mean-field terms in the energy functional are not important.
It should also be noted that the existence of BEC in two dimensions needs to be treated carefully. Initial attempts have concluded that no BEC could occur in 2D trapped gases, but recent considerations within the Hartree-FockBogoliubov approximation, [30] the density-dependent interaction strength [31], and numerical simulations [32] have established firmly the occurrence of BEC for such systems. Thus, our assumption of a $2 \mathrm{D}$ condensate at $T=0$ is justified.

\section{2D INTERACTION MODELS}

In cold atom experiments a 2D geometry is obtained by trapping the atoms in a highly anisotropic trap where the axial confinement is very tight, so that the axial potential is on the same order or larger than the chemical potentials of the two components. Within this condition, the axial widths are on the order of the oscillator lengths for the axial direction $a_{j z}=\sqrt{\hbar / m_{j} \omega_{j z}}$, with $\omega_{j z}$ being the axial trap frequency for bosons $(j=B)$ and for fermions $(j=F)$. For simplicity, here and in the following, we assume that $a_{B z}=a_{F z}=a_{z}$. The value of $a_{z}$ with respect to the modulus of the 3D scattering lengths determines whether the scattering events occur in three or in two dimensions and thus suggests how to calculate the many-body interaction potentials.

The interaction couplings $g_{B B}$ and $g_{B F}$ are determined microscopically from the effective interaction potentials (twobody scattering amplitude, $T$ matrix, etc.) in the limit of low energy and momenta. In the case of a 3D system, the scattering amplitude and $g_{B B}$ and $g_{B F}$ are constants determined by the $s$-wave scattering lengths $a_{B B}$ and $a_{B F}$. In two dimensions the scattering theory approaches give rise to a logarithmic dependence $[22,23]$. Starting from a 3D system and increasing the anisotropy (by increasing the trap frequencies in the axial direction), the geometry flattens to take a pancake shape and eventually a genuine 2D system is obtained. In the following we identify different scattering regimes depending on the relation between the axial confinement length and the scattering lengths and provide expressions for the interaction couplings in these regimes.

\section{A. Quasi-3D scattering}

In this regime, the axial oscillator length $a_{z}$ of the mixture is assumed to be larger than the modulus of $a_{B B}$ and $a_{B F}$, the $s$-wave scattering lengths for BB and BF interactions, respectively. The effective BB interaction strength can be obtained by multiplying the $3 \mathrm{D}$ value of the coupling $g_{B B}^{3 \mathrm{D}}$ $=4 \pi \hbar^{2} a / m_{B}$ with a factor $|\phi(0)|^{2}=1 / \sqrt{2 \pi} a_{z}$, with $\phi(z)$ being the axial wave function. This is obtained by assuming that the motion in the $z$ direction is frozen in the ground state of the harmonic potential with trapping frequency $\omega_{j z}$ and integrating the 3D GPE over $z$ [after multiplying with $\phi^{*}(z)$ in the spirit of taking an expectation value, the chemical potential $\mu$ gets shifted by $\left.\hbar \omega_{j z} / 2\right]$. Assuming that the profile for fermions to also be Gaussian in the $z$ direction, we apply the same idea to the BF interaction $g_{B F}^{3 \mathrm{D}}=2 \pi \hbar^{2} a / m_{B F}$, where $m_{B F}$ is the reduced mass. Thus, we obtain 


$$
g_{B B}=\frac{2 \sqrt{2 \pi} \hbar^{2}}{m_{B}} \frac{a_{B B}}{a_{z}}, \quad g_{B F}=\frac{\sqrt{2 \pi} \hbar^{2}}{m_{B F}} \frac{a_{B F}}{a_{z}},
$$

as the effective interaction couplings in the quasi-3D scattering regime.

\section{B. Strictly 2D scattering}

This regime corresponds to the limit $a_{z} \ll\left|a_{B B}\right|,\left|a_{B F}\right|$. The coupling parameter we use is from a $T$ matrix calculation $[24,25,33]$, which takes into account the many-body shift in the effective collision energy of two-condensate atoms and it becomes a self-consistent problem. Since $a_{z}<\left|a_{B B}\right|,\left|a_{B F}\right|$ the calculation is purely $2 \mathrm{D}$, and the interaction strengths do not depend on the parameters in the $z$ direction. Al Khawaja $e t$ al. [24] argue that when two-condensate atoms collide at zero momentum they both require an energy $\mu_{B}$ to be excited from the condensate and thus the many-body coupling is given by evaluating at $-2 \mu_{B}$ the two-body $T$ matrix $\left(T_{2 b}\right)$, setting $g_{B B}=\left\langle 0\left|T_{2 b}\left(-2 \mu_{B}\right)\right| 0\right\rangle$. On the other hand, Lee and Morgan [33] calculate $T_{2 b}$ at $-\mu_{B}$ arguing that this result includes the effect of quasiparticle energy spectrum of the intermediate states in the collision. Gies et al. [34] claimed that the argument of Al Khawaja et al. [24] that the excitation of a single condensate atom is associated with an energy of $-\mu_{B}$ includes the mean-field energy of initial and final states and neglects the other many-body effects on the collision which are presumably included in the result $g_{B B}$ $=\left\langle 0\left|T_{2 b}\left(-\mu_{B}\right)\right| 0\right\rangle$. With this proviso we take

$$
g_{B B}=\frac{-4 \pi \hbar^{2}}{m_{B}} \frac{1}{\ln \left(\mu_{B} m_{B} a_{B B}^{2} / 4 \hbar^{2}\right)}
$$

and, similarly,

$$
g_{B F}=\frac{-2 \pi \hbar^{2}}{m_{B F}} \frac{1}{\ln \left[\left(\mu_{B}+\mu_{F}\right) m_{B F} a_{B F}^{2} / 4 \hbar^{2}\right]},
$$

where the scattering lengths $a_{B B}=a_{B B}^{2 \mathrm{D}}$ and $a_{B F}=a_{B F}^{2 \mathrm{D}}$ are in principle 2D scattering lengths. Our choice for the 2D scattering lengths will be discussed in Sec. IV. In the case of BF interaction strength, we used the reduced mass $m_{B F}$ and made the replacement $\mu \rightarrow\left(\mu_{B}+\mu_{F}\right) / 2$. Similar considerations to write down the BF $T$ matrix were also made by Mur-Petit et al. [35].

In this regime the interaction parameters must be determined self-consistently. The way the equations are written above suggests us to solve for wave functions for given values of $g_{B B}$ and $g_{B F}$, then calculate chemical potentials

$$
\begin{aligned}
\mu_{B}= & \frac{1}{N_{B}} \int d^{2} r\left\{\frac{\hbar^{2}}{2 m_{B}}\left|\nabla \psi_{B}\right|^{2}+\frac{1}{2} m_{B} \omega_{B}^{2} r^{2}\left|\psi_{B}\right|^{2}\right. \\
& \left.+g_{B B}\left|\psi_{B}\right|^{4}+g_{B F}\left|\psi_{F}\right|^{2}\left|\psi_{B}\right|^{2}\right\}
\end{aligned}
$$

and

$$
\begin{aligned}
\mu_{F}= & \frac{1}{N_{F}} \int d^{2} r\left\{\frac{\hbar^{2}}{2 m_{F}} \lambda_{W}\left|\nabla \psi_{F}\right|^{2}+\frac{1}{2} m_{F} \omega_{F}^{2} r^{2}\left|\psi_{F}\right|^{2}\right. \\
& \left.+\frac{\hbar^{2}}{m_{F}} 2 \pi\left|\psi_{F}\right|^{4}+g_{B F}\left|\psi_{F}\right|^{2}\left|\psi_{B}\right|^{2}\right\}
\end{aligned}
$$

and check whether the expressions for $g_{B B}$ and $g_{B F}$ are satisfied. To follow a common, practice we start with initial chemical potentials, calculate $g$ 's, and then calculate chemical potentials using the obtained wave functions and require self-consistency. Note that in this regime the results do not depend on the value of $\omega_{j z}$, i.e., on the value of the anisotropy parameter $\lambda=\omega_{B z} / \omega_{B}$.

\section{Quasi-2D scattering}

When $a_{z} \geq\left|a_{B B}\right|,\left|a_{B F}\right|$ collisions are bidimensional but influenced by the $z$ direction. In this regime, which is in between the previous cases, the 2D scattering length can be expressed in terms of the 3D scattering length [36]. Substituting

$$
a_{i j}^{2 \mathrm{D}}=2 \sqrt{2} \sqrt{\frac{\pi}{B}} a_{z} e^{-\sqrt{\pi / 2}\left(a_{z} / a_{i j}^{3 \mathrm{D}}\right)}
$$

in the coupling strength expressions for strictly $2 \mathrm{D}$ regime with $B \approx 0.915$, the coupling strengths now become [33]

$$
g_{B B}=\frac{\frac{2 \sqrt{2 \pi} \hbar^{2}}{m_{B}} \frac{a_{B B}}{a_{z}}}{1+\frac{1}{\sqrt{2 \pi}} \frac{a_{B B}}{a_{z}} \ln \left(B \hbar^{2} / 2 \pi \mu_{B} m_{B} a_{z}^{2}\right)}
$$

and

$$
g_{B F}=\frac{\frac{\sqrt{2 \pi} \hbar^{2}}{m_{B F}} \frac{a_{B F}}{a_{z}}}{1+\frac{1}{\sqrt{2 \pi}} \frac{a_{B F}}{a_{z}} \ln \left[B \hbar^{2} / 2 \pi\left(\mu_{B}+\mu_{F}\right) m_{B F} a_{z}^{2}\right]} .
$$

\section{RESULTS AND DISCUSSION}

We first consider a lithium mixture with particle numbers $N_{B}=10^{6}$ and $N_{F}=5 \times 10^{5}$ and radial trapping frequencies $\omega_{B} / 2 \pi=4000 \mathrm{~Hz}$ and $\omega_{F} / 2 \pi=3520 \mathrm{~Hz}$. The $\mathrm{BB}$ and the $\mathrm{BF}$ scattering lengths are taken as $a_{B B}=5.1 a_{0}$ and $a_{B F}$ $=38 a_{0}$, respectively, in which $a_{0}$ is the Bohr radius.

In Fig. 1 we show the density distributions $n_{B}(r)$ and $n_{F}(r)$ of bosonic and fermionic components in the three scattering regimes: the quasi-3D, where the coupling is given by Eq. (5), the quasi-2D, where the coupling is given in Eqs. (11) and (12), and the strictly $2 \mathrm{D}$, where we use the coupling given in Eqs. (6) and (7) and where we set the bidimensional scattering lengths equal to $a_{i j}^{2 \mathrm{D}}$ [Eq. (10)] evaluated in the limit of vanishing $a_{z} / a_{i j}^{3 \mathrm{D}}$. This choice assures the strictly 2D model to be the limiting case of the Q2D that depicts the crossover behavior.

When $a_{B F} / a_{z}=0.1$ (top panel) the mixture has a 3D character in terms of collisions even though the geometrical con- 

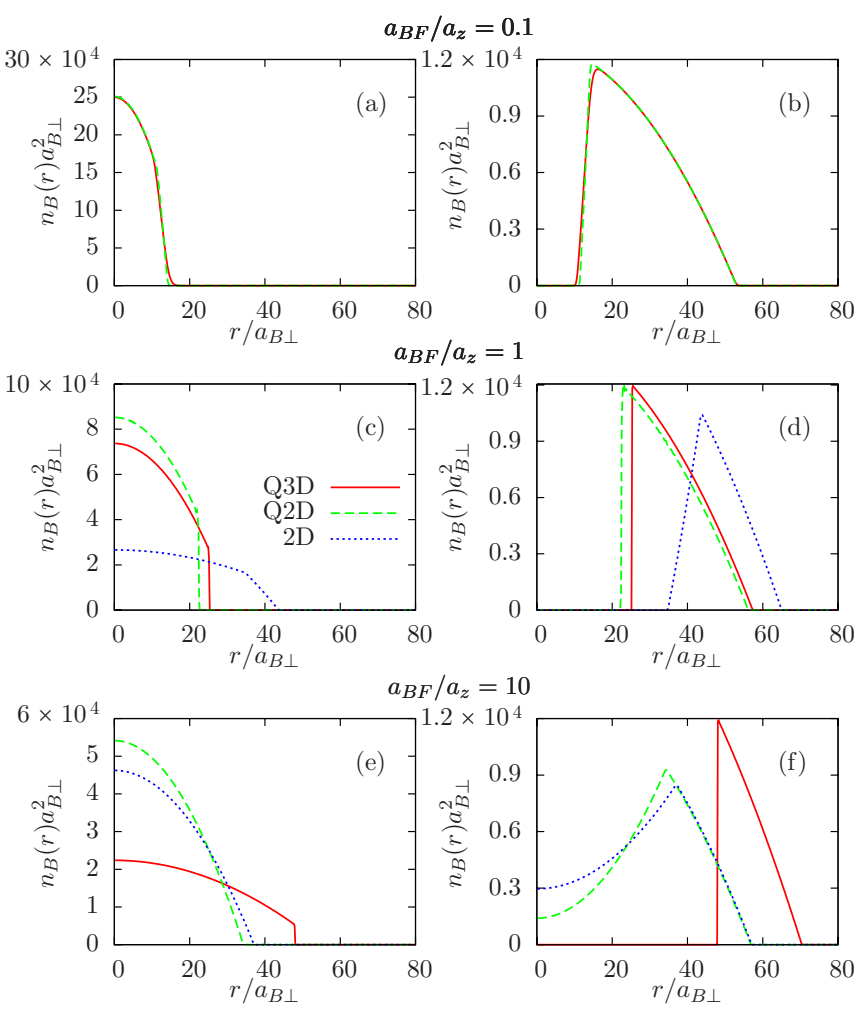

FIG. 1. (Color online) Boson and fermion density profiles for ${ }^{6} \mathrm{Li}-{ }^{7} \mathrm{Li}$ mixture with $N_{B}=10^{6}$ and $N_{F}=5 \times 10^{5}$; radial trapping frequencies $\omega_{B} / 2 \pi=4000 \mathrm{~Hz}, \omega_{F} / 2 \pi=3520 \mathrm{~Hz}$; and scattering lengths $a_{B B}=5.1 a_{0}, a_{B F}=38 a_{0}$, where $a_{0}$ is the Bohr radius. The length unit is the radial harmonic-oscillator length for bosons $a_{B \perp}$ $=\sqrt{\hbar / m_{B} \omega_{B}}$. The density given is in units of $10^{-4} a_{B \perp}^{-2}$ and is normalized to unity. The three regimes $a_{B F} / a_{z}=0.1,1,10$ correspond to values of the asymmetry parameter $\lambda \approx 10^{3}, 10^{5}$, and $10^{7}$, respectively.

finement $\left(\lambda=10^{3}\right)$ renders the system 2D kinematically. The calculated chemical potentials $\mu_{B} / \hbar \omega_{B z}$ and $\mu_{F} / \hbar \omega_{F z}$ of being less than unity also confirms that the system is geometrically 2D. In this regime the density distributions for the quasi-3D and the quasi-2D models look very similar. The boson and fermion components occupy the inner and the outer parts of the disk giving a segregated phase for the chosen parameters. The $2 \mathrm{D}$ model is evidently inapplicable in this regime because $a_{B F} / a_{z}<1$.

In the middle panels of Fig. 1 we show density profiles for the same mixture with $a_{B F} / a_{z}=1$ for an anisotropy parameter $\lambda=10^{5}$. This corresponds to a completely frozen motion in the $z$ direction and to the crossover in the scattering properties from $3 \mathrm{D}$ to $2 \mathrm{D}$. Figures $1(\mathrm{c})$ and $1(\mathrm{~d})$ reveal that the density profiles in the three models are very similar, except for the fact that the $2 \mathrm{D}$ model predicts a larger spatial extension of the density profiles.

Finally, in the bottom panel of Fig. 1 we consider $a_{B F} / a_{z}=10$ with $\lambda=10^{7}$. With $a_{z}$ being smaller than in the previous case, the bidimensional scattering lengths are smaller and both the 2D and the Q2D models predict a mixed phase even in the center of the trap, while the Q3D curves still show phase separation. For this anisotropy parameter, the scattering events should be truly $2 \mathrm{D}$ and our correspond-
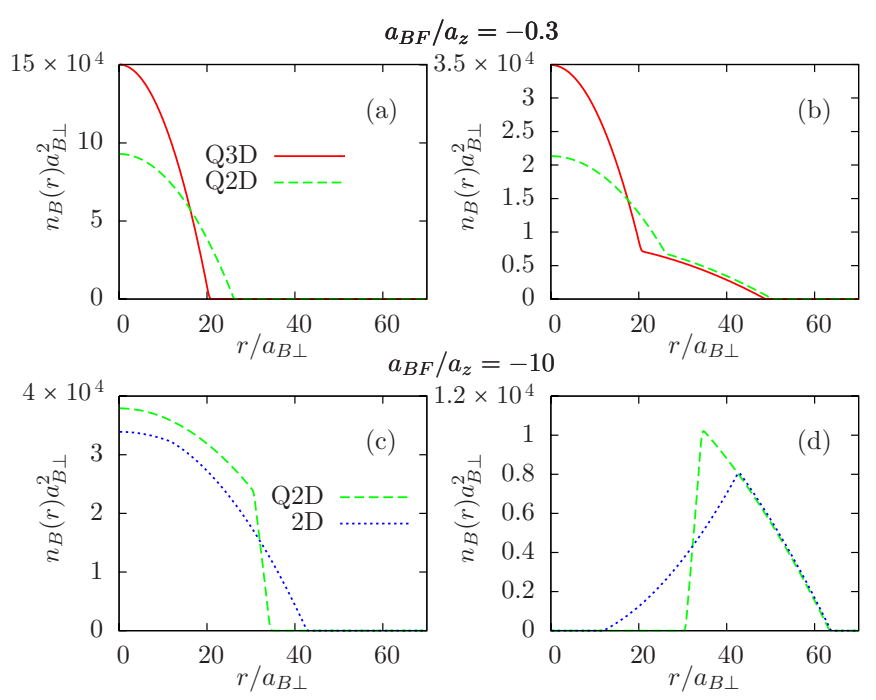

FIG. 2. (Color online) Same as in Fig. 1 for a ${ }^{40} \mathrm{~K}-{ }^{87} \mathrm{Rb}$ mixture with $N_{B}=10^{6}, N_{F}=5 \times 10^{5}$. The values of $\left|a_{B F}\right| / a_{z}=0.3,10$ correspond to $\lambda \approx 2 \times 10^{2}$ and $2 \times 10^{5}$.

ing model should yield the most accurate density profiles. Evidently the Q3D model is not yet valid, but we plot it just to compare the predictions of the different models.

We now turn our attention to ${ }^{40} \mathrm{~K}-{ }^{87} \mathrm{Rb}$ mixture having an attractive $\mathrm{BF}$ scattering length. We consider a system with particle numbers $N_{B}=10^{6}$ and $N_{F}=5 \times 10^{5}$ and radial trapping frequencies $\omega_{B} / 2 \pi=257 \mathrm{~Hz}$ and $\omega_{F} / 2 \pi=378 \mathrm{~Hz}$. The $\mathrm{BB}$ and the BF scattering lengths are taken as $a_{B B}=110 a_{0}$ and $a_{B F}=-284 a_{0}$, respectively [37]. For attractive interactions, the effective 2D BF scattering length is positive [see Eq. (10)], namely, the dimensional crossover induces effective repulsive interactions [35], as already predicted in a condensate with attractive boson-boson interaction [36]. Thus, the strictly $2 \mathrm{D}$ couplings [24,33-35] refer to hard-core collisions [22].

Figure 2 illustrates the density profiles $n_{B}(r)$ and $n_{F}(r)$ in quasi-3D and 2D scattering regimes, characterized by $a_{B F} / a_{z}=-0.3\left(\lambda \approx 2 \times 10^{2}\right)$ and $a_{B F} / a_{z}=-10\left(\lambda \approx 2 \times 10^{5}\right)$, respectively. In the case $a_{B F} / a_{z}=-0.3$, we observe that the density profiles are similar for the quasi-3D and the quasi-2D models and show a bump in the center of the fermionic density due to the attractions with the bosons. For $a_{B F} / a_{z}=-10$, the Q2D model approaches the 2D one, the only difference being that the first model predicts a complete spatial separation between the bosonic and the fermionic components, while the second predicts a residual mixed phase at the center of the trap.

The crossover between the two regimes is shown in Fig. 3. For $\lambda<10^{2}$, the fermionic density is enhanced at the center of the trap because of the presence of the bosons. In this regime the BF coupling term is negative, as shown in Fig. 4. At $\lambda=10^{5}$ the fermions are pushed out of the center of the trap because of the large repulsive BF interaction (see Fig. 4). By increasing further and further the anisotropy, the BF coupling is still positive but decreases and the two components are partially mixed. For $10^{2}<\lambda<10^{5}$ no stable solutions are found.

Thus, as shown in Fig. 4, the dimensional crossover plays the role of a Feshbach resonance. Squeezing the trap, one 

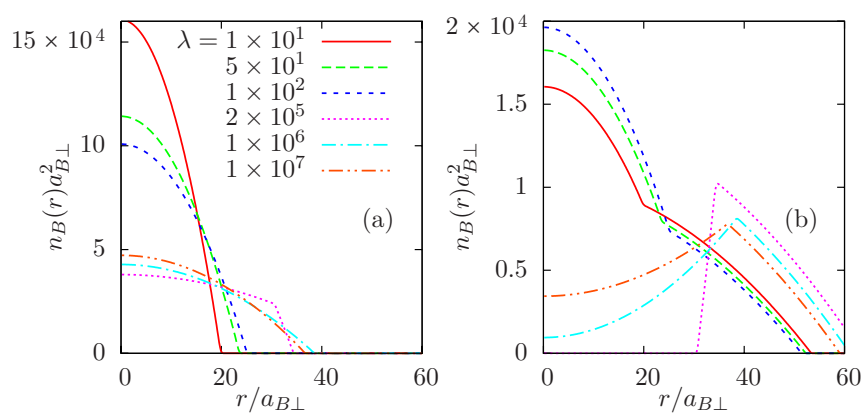

FIG. 3. (Color online) Density profiles for the ${ }^{40} \mathrm{~K}-{ }^{87} \mathrm{Rb}$ mixture calculated with the Q2D model for various values of $\lambda$ (same units as in Fig. 1).

may naively expect the gas just collapsing, but the crossover in the scattering geometry changes the nature of the instability from collapse to demixing, and a further squeezing of the trap stabilizes the mixture. All curves shown in this sections correspond to densities that fulfill the diluteness conditions $n_{B} a_{B B}^{2} \ll 1$ and $n_{F} a_{B F}^{2} \ll 1$, even at close to the resonance shown in Fig. 4.

\section{SUMMARY}

In summary we have studied the equilibrium properties of a boson-fermion mixture confined in a pancake-shaped trap, in the dimensional crossover from three to two dimensions. The boson-boson and the boson-fermion couplings used are those derived from the two-body $T$ matrix evaluated (i) at zero energy in three dimensions, (ii) taking into account the discreteness of the spectrum in the axial direction, in the crossover, and (iii) taking into account the many-body energy shift in the strictly $2 \mathrm{D}$ limit. The density profiles and the couplings have been evaluated self-consistently using suitable modified coupled Gross-Pitaevskii equations for the bosonic and the fermionic wave functions.

For the case of a positive 3D boson-fermion scattering length, the dimensional crossover softens the repulsion, so that the components of a demixed boson-fermion mixture in three dimensions can mix in the $2 \mathrm{D}$ limit. For the case of a

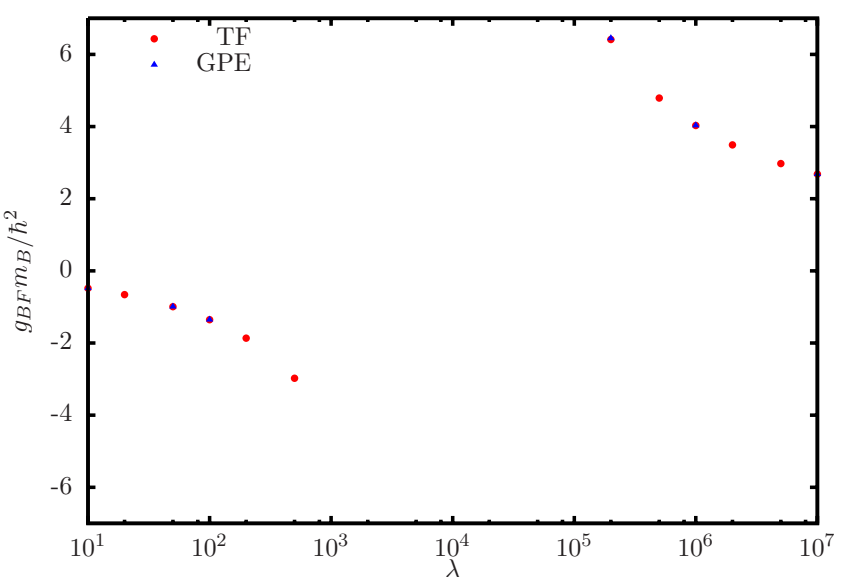

FIG. 4. (Color online) Effective BF interaction strength (in dimensionless units) for the ${ }^{40} \mathrm{~K}-{ }^{87} \mathrm{Rb}$ mixture within the Q2D model as a function of the anisotropy parameter $\lambda$. Dots refer to the numerical calculation performed in the TF approximation, namely, neglecting the Laplacian terms in Eqs. (3) and (4), while triangles refer to the full solution of the same equations (GPE).

negative 3D boson-fermion scattering length, the dimensional crossover is more dramatic and plays the role of a Feshbach resonance. Our study shows that the squeezing of the pancake-shaped trap may drive a strong-attractive unstable mixture toward a stable mixed mixture passing through a demixed phase. This numerical study may be reproduced in the actual experiments with BF mixtures. With the goal of being able to reach a regime where the modulus of the scattering lengths is comparable or greater than the mixture axial size, one may exploit Feschbach resonances to increase the magnitude of the 3D scattering lengths, or one may engineer very flat traps as already done in the context of experiments with a single BEC component.

\section{ACKNOWLEDGMENTS}

This work was supported by TUBITAK (Grant No. 108T743), TUBA, and European Union 7th Framework Project UNAM-REGPOT (Grant No. 203953).
[1] A. G. Truscott, K. E. Strecker, W. I. McAlexander, G. B. Partridge, and R. G. Hulet, Science 291, 2570 (2001).

[2] F. Schreck, L. Khaykovich, K. L. Corwin, G. Ferrari, T. Bourdel, J. Cubizolles, and C. Salomon, Phys. Rev. Lett. 87, 080403 (2001).

[3] Z. Hadzibabic, C. A. Stan, K. Dieckmann, S. Gupta, M. W. Zwierlein, A. Görlitz, and W. Ketterle, Phys. Rev. Lett. 88, 160401 (2002).

[4] J. Goldwin, S. B. Papp, B. DeMarco, and D. S. Jin, Phys. Rev. A 65, 021402(R) (2002).

[5] G. Roati, F. Riboli, G. Modugno, and M. Inguscio, Phys. Rev. Lett. 89, 150403 (2002).

[6] G. Modugno, G. Roati, F. Riboli, F. Ferlaino, R. J. Brecha, and M. Inguscio, Science 297, 2240 (2002).
[7] T. Fukuhara, S. Sugawa, Y. Takasu, and Y. Takahashi, Phys. Rev. A 79, 021601(R) (2009).

[8] G. B. Partridge, W. Li, R. I. Kamar, Y. Liao, and R. G. Hulet, Science 311, 503 (2006).

[9] Y. Shin, M. W. Zwierlein, C. H. Schunck, A. Schirotzek, and W. Ketterle, Phys. Rev. Lett. 97, 030401 (2006).

[10] S. Ospelkaus, C. Ospelkaus, L. Humbert, K. Sengstock, and K. Bongs, Phys. Rev. Lett. 97, 120403 (2006).

[11] M. Zaccanti, C. D’Errico, F. Ferlaino, G. Roati, M. Inguscio, and G. Modugno, Phys. Rev. A 74, 041605(R) (2006).

[12] K. Mølmer, Phys. Rev. Lett. 80, 1804 (1998).

[13] Z. Akdeniz, P. Vignolo, A. Minguzzi, and M. P. Tosi, J. Phys. B 35, L105 (2002).

[14] R. Roth, Phys. Rev. A 66, 013614 (2002). 
[15] S. Röthel and A. Pelster, Eur. Phys. J. B 59, 343 (2007).

[16] Z. Akdeniz, P. Vignolo, and M. P. Tosi, Phys. Lett. A 331, 258 (2004).

[17] A. Görlitz, J. M. Vogels, A. E. Leanhardt, C. Raman, T. L. Gustavson, J. R. Abo-Shaeer, A. P. Chikkatur, S. Gupta, S. Inouye, T. Rosenband, and W. Ketterle, Phys. Rev. Lett. 87, 130402 (2001).

[18] V. Schweikhard, I. Coddington, P. Engels, V. P. Mogendorff, and E. A. Cornell, Phys. Rev. Lett. 92, 040404 (2004).

[19] D. Rychtarik, B. Engeser, H.-C. Nägerl, and R. Grimm, Phys. Rev. Lett. 92, 173003 (2004).

[20] Y. Colombe, E. Knyazchyan, O. Morizot, B. Mercier, V. Lorent, and H. Perrin, EPL 67, 593 (2004).

[21] S. Stock, Z. Hadzibabic, B. Battelier, M. Cheneau, and J. Dalibard, Phys. Rev. Lett. 95, 190403 (2005).

[22] M. Schick, Phys. Rev. A 3, 1067 (1971).

[23] V. N. Popov, Functional Integrals in Quantum Field Theory and Statistical Physics (Reidel, Dordrecht, 1983), Chap. 6.

[24] U. Al Khawaja, J. O. Andersen, N. P. Proukakis, and H. T. C. Stoof, Phys. Rev. A 66, 013615 (2002).

[25] M. D. Lee, S. A. Morgan, M. J. Davis, and K. Burnett, Phys. Rev. A 65, 043617 (2002).

[26] B. Tanatar, A. Minguzzi, P. Vignolo, and M. P. Tosi, Phys. Lett. A 302, 131 (2002).
[27] O. Hosten, P. Vignolo, A. Minguzzi, B. Tanatar, and M. P. Tosi, J. Phys. B 36, 2455 (2003).

[28] See, for instance, B. P. van Zyl and E. Zaremba, Phys. Rev. B 59, 2079 (1999).

[29] D. M. Jezek, M. Barranco, M. Guilleumas, R. Mayol, and M. Pi, Phys. Rev. A 70, 043630 (2004); M. E. Tasgin, A. L. Subasi, M. O. Oktel, and B. Tanatar, J. Low Temp. Phys. 138, 611 (2005).

[30] C. Gies, B. P. van Zyl, S. A. Morgan, and D. A. W. Hutchinson, Phys. Rev. A 69, 023616 (2004).

[31] B. P. van Zyl, R. K. Bhaduri, and J. Sigetich, J. Phys. B 35, 1251 (2002).

[32] M. Holzmann and W. Krauth, Phys. Rev. Lett. 100, 190402 (2008).

[33] M. D. Lee and S. A. Morgan, J. Phys. B 35, 3009 (2002).

[34] C. Gies, M. D. Lee, and D. A. W. Hutchinson, J. Phys. B 38, 1797 (2005).

[35] J. Mur-Petit, A. Polls, M. Baldo, and H.-J. Schulze, Phys. Rev. A 69, 023606 (2004); J. Phys. B 37, S165 (2004).

[36] D. S. Petrov and G. V. Shlyapnikov, Phys. Rev. A 64, 012706 (2001).

[37] C. Ospelkaus, S. Ospelkaus, K. Sengstock, and K. Bongs, Phys. Rev. Lett. 96, 020401 (2006). 